\title{
Uranium blocks for cylindrical projections of the critical radius
}

\author{
Li Wei \\ School of physics, Baicheng Normal college \\ JiLin Baicheng, China, liweiliwei77@163.com
}

Keywords: cylindrical uranium; zero-order Bessel equation; boundary conditions; definite solution of the problem; critical radius

Abstract. In this paper, mathematical physics equations Bessel equation solving means, for the critical radius cylindrical uranium blocks were theoretically derived and obtained relevant results. Uranium radioactive material, there are two forms of exercise neutrons, neutron multiplication neutron diffusion and movement, through experimental tests, per unit volume in the number of neutrons produced is proportional to the neutron density $\mathrm{S}$.

\section{Introduction}

Uranium radioactive material, there are two forms of exercise neutrons, neutron multiplication neutron diffusion and movement, through experimental tests, per unit volume in the number of neutrons produced is proportional to the neutron density $\mathrm{u}$,

Because there is a diffusion source proliferative response, proportional to the concentration and diffusion source, get the diffusion equation :

$$
u_{t}-a^{2} \Delta u=\beta u
$$

Expression in the meaning of the variables:

$a^{2}-$ The diffusion coefficient;

$\beta-$ Neutron multiplication constant movement.

Through description of the problem, we find that it is difficult to use conventional methods to solve this problem. we try to approach in cylindrical coordinates, first of all, we want to establish a process of problem solving in cylindrical coordinates. Assuming cylindrical coordinates Laplace equation operator expression (2):

$$
\frac{1}{\rho} \frac{\partial}{\partial \rho}\left(\rho \frac{\partial u}{\partial \rho}\right)+\frac{1}{\rho^{2}} \frac{\partial^{2} u}{\partial \theta^{2}}+\frac{\partial^{2} u}{\partial z^{2}}=0
$$

Laplace equation above problems can be described in Figure 1:

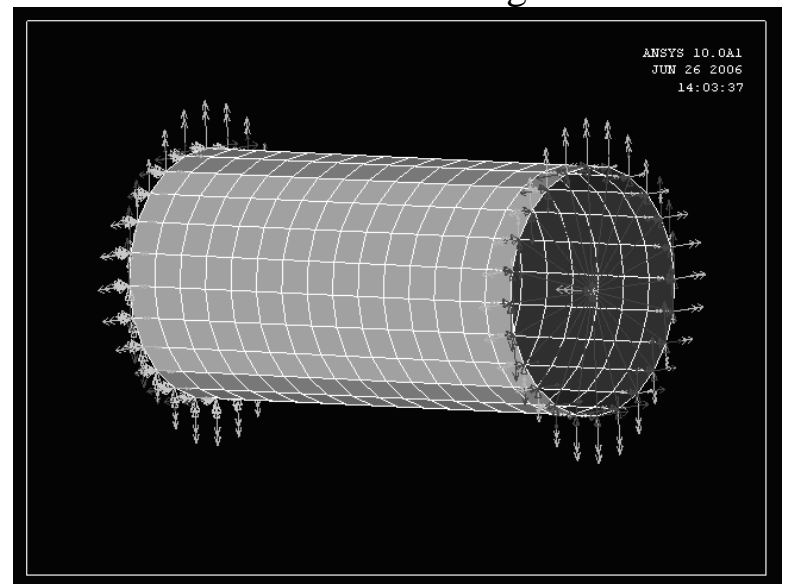

Figure 1. Cylindrical coordinates Laplace equation

Separated variables for $u$ : $u(\rho, \varphi, z)=R(\rho) \Phi(\varphi) Z(z)$

The results are substituted into the expression (1) can be obtained: 
$\Phi Z\left(\frac{d^{2} R}{d \rho^{2}}\right)+\frac{\Phi Z}{\rho} \frac{d R}{d \rho}+\frac{R Z}{\rho^{2}} \Phi^{\prime \prime}+R \Phi Z^{\prime \prime}=0$

The expression (1) for variable collation, we can get equations:

$$
\left\{\begin{array}{l}
\Phi^{\prime \prime}+\lambda \Phi=0 \\
\frac{\rho^{2}}{R} \frac{d^{2} R}{d \rho^{2}}+\frac{\rho}{R} \frac{d R}{d \rho}+\rho^{2} \frac{Z^{\prime \prime}}{Z}=\lambda
\end{array}\right.
$$

For this eigenvalue problem is solved: $\lambda=m^{2} \quad(m=0,1,2 \ldots)$,

Eigenvalues expression is the expression (5):

$$
\Phi(\varphi)=A \cos m \varphi+B \sin m \varphi
$$

$\lambda=m^{2}$ substituted into the expression (4), while the expression of both sides of the equation is divided by $\left(1 / \rho^{2}\right)$ :

$$
\frac{1}{R} \frac{d^{2} R}{d \rho^{2}}+\frac{1}{\rho R} \frac{d R}{d \rho}-\frac{m^{2}}{\rho^{2}}=\frac{-Z^{\prime \prime}}{Z}
$$

Clearly, the expression on the left contains only the variable $\mathrm{Z}$, expression on the right contains only the variable $\rho$, if the equality holds, the left and right of the expression must be equal to the same constant expressions:

$$
\left\{\begin{array}{l}
Z^{\prime \prime}-\mu Z=0 \\
\frac{d^{2} R}{d \rho^{2}}+\frac{1}{\rho} \frac{d R}{d \rho}+\left(\mu-\frac{m^{2}}{\rho^{2}}\right) R=0
\end{array}\right.
$$

The next task is to discuss the intrinsic value $\mu$ :

1. when $\mu=0$, After calculation, the equation can be obtained (7) of the root:

$Z=C+D z$

Obtain equation (8) in the root:

$$
R= \begin{cases}E+F \ln \rho & (m=0) \\ E \rho^{m}+F / \rho^{m} & (m=1,2 \ldots)\end{cases}
$$

2. When $\mu>0$, Replace the variable: $x=\sqrt{\mu} \rho$, substituted into the expression (8), the new expression (8) :

$\frac{d R}{d \rho}=\frac{d R}{d x} \frac{d x}{d \rho}=\sqrt{\mu} \frac{d R}{d x}$

The expression (8) to make adjustments:

$x^{2} \frac{d^{2} R}{d x^{2}}+x \frac{d R}{d x}+\left(x^{2}-m^{2}\right) R=0$

Equation (7) The roots of the expression (14):

$$
Z(z)=C e^{\sqrt{\mu} z}+D e^{-\sqrt{\mu} z}
$$

3. When $\mu<0$, the equation (7) is the root of the expression :

$$
Z(z)=C \cos v z+D \sin v z
$$

On the equation (8) in the variable $\mathrm{x}$ to do substitution: $x=v \rho$, equation (8) of the new style:

$\frac{d^{2} R}{d x^{2}}+\frac{d R}{x d x}-\left(1+\frac{m^{2}}{x^{2}}\right) R=0$

Obtain equation : 


$$
x^{2} \frac{d^{2} R}{d x^{2}}+x \frac{d R}{d x}-\left(m^{2}+x^{2}\right) R=0
$$

Cylindrical coordinates Laplace equation solving process can be described in Figure 2.

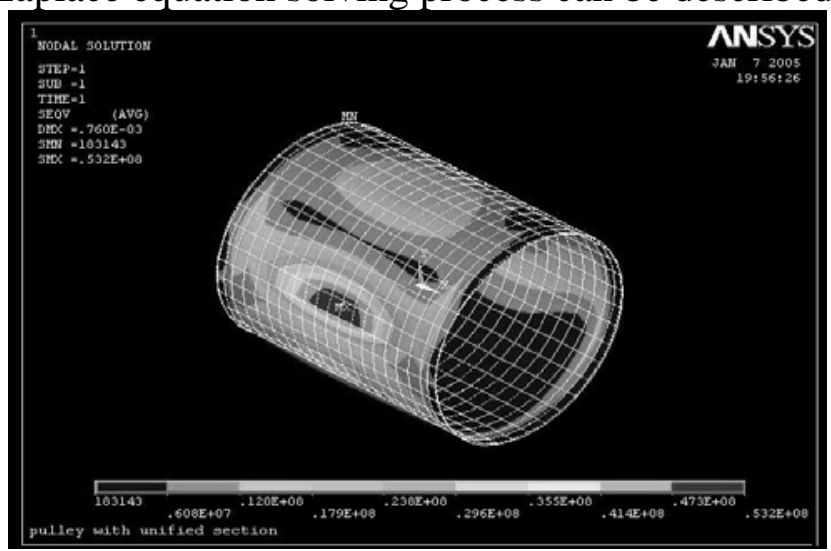

Figure 2. Cylindrical coordinates Laplace equation

We assume that the radius of the uranium column is $\mathrm{R}$, therefore, the establishment of a condition of $\left.u\right|_{\rho=R}=0$. Because it is a long cylindrical, our problem has nothing to do with variables $\mathrm{z}$. simultaneously, z-axis is the axis of symmetry, this problem has nothing to do with variables $\varphi$, so we got in a cylindrical coordinate system made up Solutions of equations:

$$
\left\{\begin{array}{l}
u_{t}-a^{2}\left(u_{\rho \rho}+\frac{u_{p}}{\rho}\right)=\beta u \\
\left.u\right|_{\rho=R}=0
\end{array}\right.
$$

In cylindrical coordinates, the problem can be described in Figure 3:

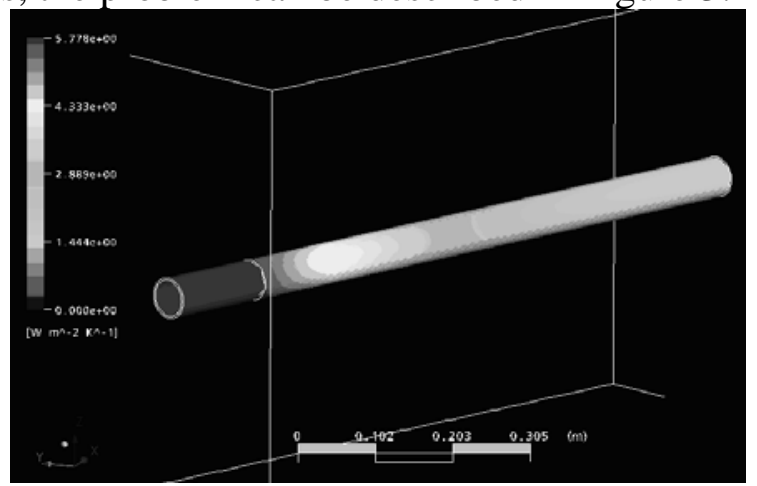

Figure 3. Solution of the problem and the boundary conditions

Equation for the implementation of separation of variables, Obtain expressions:

$$
\left\{\begin{array}{l}
T=A e^{\lambda t} \\
u^{\prime \prime}+\frac{v^{\prime}}{\rho}+\frac{(\beta-\lambda)}{a^{2}} v=0 \\
\left.v\right|_{\rho=R}=0
\end{array}\right.
$$

Equation (21) is the zero order Bessel equation, It is the body of the column there is a finite root: $v(\rho)=B J_{0}\left(\frac{\sqrt{\beta-\lambda}}{\alpha} \rho\right)$. 
Expression can be obtained through the boundary conditions: $J_{0}\left(\frac{\sqrt{\beta-\lambda}}{\alpha} \rho\right)=0$, Because ${ }^{x_{n}^{(0)}}$ is $J_{0}(x)$ the n pole of order, Means $x_{n}^{(0)}=\frac{\sqrt{\beta-\lambda_{n}^{(0)}}}{\alpha} R$.

In cylindrical coordinates, the problem solving process can be described in Figure 4:

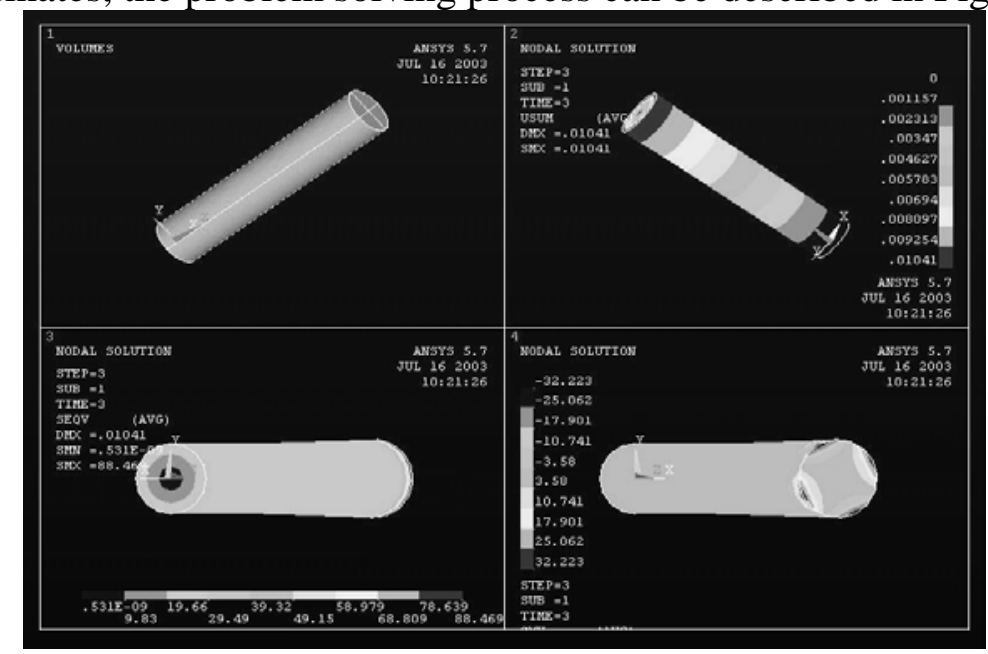

Figure 4. Roots of the equation, in cylindrical coordinate system

\section{Summary}

By Equation (20) can be obtained the following conclusions:

When $\beta>0$, if the index contains $\lambda_{n}^{(0)}>0$ items, the cause of the event $\beta>\left(\frac{a x_{n}^{(0)}}{R}\right)^{2}$. With the increase of variable t. exponentially increasing the concentration of neutrons, the reactor will go broke state.

When $\beta-\left(\frac{a x_{n}^{(0)}}{R}\right)^{2}=0, x_{n}^{(0)}$ pole there is a zero-order $x_{n}^{(1)}=2.4048$, By solving the equation, the critical radius cylindrical uranium blocks expression: $R_{k p}=\frac{2.4048 a}{\sqrt{\beta}}$.

\section{References}

[1] Black L;Miserque F; Gouder T Preparation and photoelectron spectroscopy study of UN thin films 2007(2).

[2] Vaquila I;Vergara L I;Passeggi M C G Chemical Reaction at Surface: Titanium Oxidation 1999(1).

[3] Gang Lu;Steven L;Bernasek;Jeffrey Schwartz Oxidation of a Polycrystalline Titanium Surface by Oxygen and Water 2000.

[4] Gunasekhar K R;Srinivasulu S;Swarnalatha M Structure and Microstructure of Ion-Plated Titanium Films 1994.

[5] Martin M;Mader W;Fromm E Oxidation of Iron, Aluminum and Titanium Films in the Temperature Range 50-200 